Reichard: Ueber d. Nachw. d. Chromsäure durch Wasserstofisuperoxyd etc. 577

I. Nach der Formel entspricht $1 g$ Permanganat also:

$$
\begin{array}{lc}
0,5883 g & \text { Natriumhyposulfit, } \\
0,4553 \ll & \mathrm{SO}_{4}, \\
0,3478 \ll & \cdot \mathrm{Mn} .
\end{array}
$$

II. Nach der quantitativen Analyse, auf $1 \mathrm{~g}$ Permanganat berechnet (vergleiche b, quantitative Prüfung):

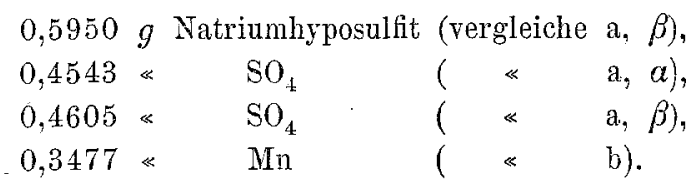

Es ergibt sich hieraus, dass Sulfatformel und Analyse so ziemlich stimmen. Die Differenzen stammen aller Wahrscheinlichkeit nach von einer Nebenreaction her. (Siehe $\approx$ Qualitative Prüfung «, 2 und 3.)

Ueber den Nachweis der Chromsänre durch Wasserstoffsuperoxyd bei Gegenwart von Vanadinsänre.

$$
\text { Von }
$$

\title{
C. Reichard.
}

Eine der schärfsten, wenn nicht die schärfste Reaction zum Nachweise der Chromsäure and ihrer Salze besteht bekanntlich in der Einwirkung von Wasserstoffsuperoxyd auf die genannte Verbindung bei Anwesenheit von Säuren. Es tritt eine Blaufärbung auf, welche durch die Bildung von Ueberchromsäure $\left(\mathrm{Cr}_{2} \mathrm{O}_{7}\right)$ hervorgerufen wird. Letztere Säure besitzt die Eigenthümlichkeit, analog einigen complexen Säuren, sich in Aether, und zwar mit intensiv blaner Farbe, zu lösen. Ueberlässt man die ätherische Lösung sich selbst bei ungehindertem Zutritt der atmosphärischen Luft, so tritt nach einiger Zeit Reduction der Ueberchromsäure ein unter Entfärbung des Aethers. Wahrscheinlich wird diese Entfärbung durch die organischen, in der Atmosphäre befindlichen Materien verursacht. Nach Storer ist die Empfindlichkeit der Ueberchromsäure-Reaction besonders bei Zusatz von Aether so bedeutend, dass noch 1 Theil chromsaures Kalium, welches in 40000 Theilen Wasser gelöst ist, eine wahrnehmbare Blaufürbung liefert. Diese Verdünnung stellt eine Lösung dar, welche in 100 Theilen Wasser $0,0025 \mathrm{~g}$ Kaliumchromat enthält. Ist neben der Chromsäure gleichzeitig Vanadin- 
säure oder ein Salz derselben vorhanden, so wird die Empfindlichkeit der durch Wasserstoffsuperoxyd bewirkten Reaction nach den Angaben von Werther ${ }^{1}$ ) wesentlich beeinflusst, das heisst beeinträchtigt. In Folge dieser Beobachtungen sah ich mich veranlasst, nach Möglichkeit die Grenze festzustellen, welche der Wasserstoffsuperoxyd-Reaction gezogen ist bei Gegenwart von vanadinsauren Salzen, und zugleich das Verhalten der Ueberchromsänre bei Anwesenheit ron Vanadinsäure verschiedenen anderen chemischen Einflüssen gegenüber näher zu untersuchen.

Von der Erwägung ausgehend, dass man bei der ausserordentlichen Schärfe der Reaction zwischen Chromsäure and Wasserstoffsuperoxyd sich dieser Reaction speciell beim Nachweise sehr geringer Mengen von chromsauren Salzen bedienen wird, operirte ich mit solchen Lösungen, welche $0,1 \%$ an löslichem Kaliumbichromat enthielten. Die Lösung der Vanadinsäure wurde in der Concentration hergestellt, dass in $100 c c 0,1 g$ metavanadinsaures Ammoniak gelöst war. Das Ammoniummetavanadat wurde aus dem Grunde bevorzugt, weil es sich in grosser Reinheit aus seinen Lösungen durch eine Auflösung von Chlorammonium in Wasser abscheiden lässt.

Was die von Werther ${ }^{2}$ ) behauptete Beeinträchtigung der Empfindlichkeit der Wasserstoffsuperoxyd-Reaction durch Vanadinsäure angeht, so kann sie entweder darauf zurückgeführt werden, dass durch die Gegenwart der Vanadinsäure eine Reduction der Ueberchromsäure stattfindet und erstere Säure ebenfalls eine Veränderung erleidet, oder darauf, dass die Vanadinsäure ein ihr eigenthümliches Vermögen besitzt, die Intensität der Ueberchromsäure-Reaction $\mathrm{zu}$ beeinträchtigen, dabei sich aber selbst passiv verhält. Im ersteren Falle lässt sich erwarten, dass ein grösserer Zusatz von Wasserstoffsuperoxyd, als zur Oxydation dér vorhandenen Chromsäure erforderlich ist, die durch Vanadinsäure eventuell beeinträchtigte Intensität der Blaufärbung wieder herstellen wird. Im zweiten Falle würde man sich die Einwirkung der Vanadinsäure so etwa vorzustellen haben wie beispielsweise die des Mangansuperoxyds, des Kupferoxyds und so weiter auf Lösungen von Chlorkalk; in dem einen Fall würde die Ueberchromsäure, im anderen die unterchlorige Säure zerstört durch Entwicklung von Sauerstoff. Trifft die

1) Journ. f. prakt. Chemie $83,195$.

2) A. a. 0 . 
erstere Vermuthung $z \mathfrak{u}$, so kann von einer Beeinträchtigung der Wasserstoffsuperoxyd-Reaction durch Vanadinsäure, wie Werther behauptet, überhaupt nicht die Rede sein, da es ja frei steht, deren möglichen Einfluss durch vermehrten Zusatz von Wasserstoffsuperoxyd zu reguliren, beziehungsweise aufzuheben. Nach meinen Erfahrungen ist der zweiten Möglichkeit der Vorzug zu geben. Die bei den einzelnen Versuchen auftretenden Erscheinungen lassen darauf schliessen, dass die Vanadinsäure keine Zersetzung erleidet. Vorausschicken will ich vor Besprechung der einzelnen von mir vorgenommenen Versuche, dass die Vanadinsäure (in Form ihres Meta-Ammoniumsalzes) einen unverkennbar bedeutenden Einfluss sowohl auf die bereits dargestellte Ueberchromsäure, als auf deren Erzeugung besitzt, and dass in der That selbst bei Gegenwart von relativ kleinen Mengen von metavanadinsaurem Ammoniak verhältnissmässig bedeutende Mengen von Chromsäure völlig passiv bleiben können, wenn man sie in bekannter Weise in die höchste Sauerstoffverbindung des Chroms überzuführen beabsichtigt.

Zum ersten Versuch wurden $3 c c$ Kaliumbichromatlösung - enthaltend $0,003 \mathrm{~g} \mathrm{~K}_{2} \mathrm{Cr}_{2} \mathrm{O}_{7}$ - mit $3 \mathrm{cc}$ einer etwa 3 procentigen Wasserstoffsuperoxydlösung geschüttelt, and 1 Tropfen einer 25 procentigen Salzsäure hinzugefügt. Durch den Einfluss der Säure nahm die Lösung eine tief dunkelblane Färbung an, welche nach einiger Zeit bei ruhigem Stehen allmählich in Hellblau überging. Wurden die gleichen Verhältnisse gewählt wie eben, nur mit dem Unterschiede, dass der gerade blau gewordenen Flüssigkeit $2 c c$ einer Lösung von meta-vanadinsaurem Ammoniak hinzugefügt wurden (entsprechend 0,002 $g$ meta-vanadinsaurem Ammon), so trat nach wenigen Augenblicken eine Entfärbung der dunkelblauen Flüssigkeit ein, indem an deren Stelle eine bräunlich gefärbte entstand. In diesem Falle verhielt sich das anwesende Kaliumbichromat zu dem vanadinsauren Ammoniak wie $3: 2$. Aber auch, wenn das Verhältniss sich stellte wie $3: 1$, war deutlich und in der gleichen Zeit der nämliche Effect $z u$ beobachten. Auf jeden Fall geht bereits aus den minimalen Mengen - landelte es sich doch auf beiden Seiten nur um Milligramme - die zur Anwendung gelangten, hervor, dass die Vanadinsäure in hohem Grade die Fähigkeit besitzt, die Ueberchromsäure-Reaction zu beeinträchtigen. Es sei hier bemerkt, dass die ohne Anwendung ron Vanadinsäure hervorgerufene Blaufärbung nach ihrer freiwilligen Entfärbung nicht mehr durch einen noch so grossen Zusatz von Wasserstoffsuperoxyd unter Beifügung von Salzsäure regenerirt 
werden kann. Auch Natronlauge oder Ammoniak bewirken in der schliesslich scbmułzig gelb-grau aussehenden Zersetzungsiösung der Ueberchromsäure-Reaction keine Veränderung. Wasserstoff entwickelnde Flüssigkeiten, wie zum Beispiel verdünnte Salzsäure, in welcher man Zink suspendirt hat, beschleunigen an sich den Reductionsprocess der Ueberchromsäure, ändern indessen an der bräunlichen Lösung, welche durch meta-vanadinsaures Ammoniak verursacht wurde, nichts. Modificirt man den Versuch mit dem letzt genannten Körper derart, dass bei Einhaltung der oben angegebenen Bedingungen nur die Hälfte des vanadinsauren Ammoniaks hinzugefügt wird, lässt man also auf ein Gemenge von je $3 c c$ Kaliumbichromat- und Wasserstoftsuperoxydlösung nur $0,5 c c$ metavanadinsaures Ammoniak einwirken, so ist zu bemerken, dass sich die Bräunlichfärbung zwar einstellt, dass sich dieselbe aber im Gegensatz zu den ersteren Versuchen bedeutend verzögert. Noch deutlicher als bei diesem Verhältnisse $\left(6 \mathrm{~K}_{2} \mathrm{Cr}_{2} \mathrm{O}_{7}: 1\right.$ metavanadinsaurem Ammon) lässt sich die beschriebene Thatsache beobachten, wenn man auf $9 \mathrm{ce}$ Kaliumbichromatlösung (entsprechend $0,009 \mathrm{~g} \mathrm{~K}_{2} \mathrm{Cr}_{2} \mathrm{O}_{7}$ ) $1 \mathrm{cc}$ metavanadinsaures Ammoniak ( $=0,001 \mathrm{~g}$ metavanadinsaures Ammon) einwirken lässt. Es findet kaum merkliche Einwirkung auf die Blaufärbung statt, und erst nach verhältnissmässig sehr langer Zeit tritt die erwähnte bräunliche Färbung und dazu noch in schwachem Maasse ein.

Werden einige Cubikcentimeter der 0,1 procentigen Kaliumbichromatlösung mit dem gleichen Volumen Wasserstoffsuperoxyd und 90 procentigem Alkohol unter Salzsäurezusatz behandelt, so erhält man eine ebenfalls tief dunkelblane, alkoholische Flüssigkeit; bei ruhigem Stehen an offener Luft verblasst die blaue Iösung schon innerhalb $1 / 4$ Stunde, und die Flüssigkeit wird fast farblos. Durch Natronhydrat oder Ammoniak wird die blaue Lösung sogleich entfärbt, das heisst sie nimmt eine grünlich-gelbe Tönung an. Durch Ansäuern mit Salzsäure erscheint die durch Alkalien verschwundene Blaufärbung wieder. Wie bereits erwähnt, können solche Lösungen, welche anfangs tief blau waren und sich freiwillig entfärbt haben, durch erneuten Zusatz von Wasserstoffsuperoxyd und Salzsäure nicht wieder wie ursprünglich blau gefärbt werden. Wird aber die entfärbte Flüssigkeit zuerst mit Natronlauge, dann mit Wasserstoffsuperoxyd behandelt und mit Salzsäure schwach übersättigt, so tritt wieder die ursprüngliche blaue Farbe auf, wenn auch nicht gerade so intensiv wie vorher. 
War in den bisher besprochenen Versuchen das Kaliumbichromat gegenüber dem metavanadinsauren Ammoniak im Ueberschuss zur Anwendung gekommen, so sollen im Folgenden auch die Ergebnisse einiger Versuche in umgekehrter Richtung erwähnt werden, obgleich das Resultat von vornherein nicht zweifelhaft sein konnte. Der erste Versuch war so angeordnet, dass eine Lösung von $1 c c$ Kaliumbichromatlösung (entsprechend $0,001 g \quad \mathrm{~K}_{2} \mathrm{Cr}_{2} \mathrm{O}_{\tau}$ ) mit $2 c c$ Wasserstofisuperoxyd gemischt und diese Flüssigkeit zu $5 c c$ einer mit 2 Tropfen Salzsäure versetzten Ammoniumvanadatlösung (enthaltend $0,005 \mathrm{~g}$ Ammoniumvanadat) hinzugefügt wurde. Es entstand eine bläulich grüne Färbung, die aber schon innerlalb kaum einer Minute verschwand und einer schmutzig gelbgrünen Farbe Platz machte. Modificirt man die Reaction so, dass man 5 cc Vanadatlösung mit $1 c c$ Kaliumbichromatlösung schüttelt, 2 Tropfen Salzsäure hinzufügt and nun erst $2 c c$ Wasserstoffsuperoxyd auf das Gemisch einwirken lässt, so tritt überhaupt keine Blaufärbung auf, während sie doch durch die bläulich-grüne des vorhergehenden Versuchs wenigstens angedeutet war. Die Vanadinsäurelösung wirkt also unter diesen Umständen vollkommen prohibitiv auf die Ueberchromsäure-Reaction. Die schliesslich resultirende Endfärbung ist im Uebrigen die nämliche wie vorher, nämilch eine schmutzig gelbgrüne. Bemerkt muss an dieser Stelle werden, dass die Ammoniummetavanadatlüsung, welche sonst farblos erscheint, bei dem kleinsten Zusatze von Salzsäure eine gelbliche, dem gelösten Kaliumchromat nicht unähnliche Färbung annimmt. Der Grund dafür ist wohl in der Ausscheidung von Vanadinsäure zu suchen. Wie besonders aus der Anordnung des eben beschriebenen zweiten Versuches erhellt, übt die Vanadinsäure als solche einen so bedeutenden Einfluss auf die Chromsäure aus, dass eine Einwirkung des Wasserstoffsuperoxyds überhaupt nicht mehr möglich ist. Vielleicht entsteht eine complexe Chrom-Vanadinsäure, deren Constitution eine Ueberführung des einen Componenten in Ueberchromsäure ausschliesst; vielleicht auch erleidet die Chromsäure eine Zersetzung, so dass auch in diesem Falle ein Versuch mit Wasserstoffsuperoxyd resultatlos bleiben muss.

Bekanntlich ist die Ueberchromsäure mit schön dunkelblauer Farbe in Aether löslich; wird eine wässerige Lösung mit der genügenden Menge Aether geschüttelt, so wird sie farblos, während alle färbende Materie von dem Aether aufgenommen wird, Diese ätherische Lösung verhält sich in mancher Beziehung anders als die wässerige. Während 
letztere nach einiger Zeit ruhigen Stehens eine hellblane Färbung annimmt, ist die erstere mehr beständig und behält ihre dunkle Farbe länger bei. Wird eine ätherische Lösung der Ueberchromsäure (erhalten aus je $\breve{a} c c$ einer 0,1 procentigen Kaliumbichromat-Lösung und Wasserstoffsuperoxyd) mit $1 / 2$ bis $1 c c$ einer ebenfalls 0,1 procentigen Lösung von metavanadinsaurem Ammoniak unter Zusatz von einigen Cubikcentimetern Wasser geschüttelt, so entfärbt sich die Aetherschicht und wird völlig farblos. Setzt man zu diesem farblos gewordenen Aether neuerdings etwas Wasserstoffsuperoxyd and 1 Tropfen Salzsäure und schüttelt, so erscheint die blaue Farbe des Aethers wieder, während die darunter befindliche wässerige Lösung sich bräunlich färbt. Bringt man zu der ätherischen Lösung der Ueberchromsäure einige Zinkstückchen, so entfärbt sie sich dadurch ganz langsam, ohne dass eine specielle Wasserstoffentwicklung $z u$ beobachten wäre. Man erhält auch in diesem Falle zuletzt eine total farblose Flüssigkeit. Wird eine ätherische Ueberchromsäurelösung mit Natronlange geschüttelt, so entfärbt sie sich; die farblose Aetherschicht wird durch Salzsäure schnell vorübergehend bläulich gefärbt. Setzt man das Schütteln mit Natronlauge etwas länger fort, so bringt auch ein Zusatz von Wasserstoffsuperoxyd in Verbindung mit 1 Tropfen Salzsäure im Gegensatz zu dem weiter oben erwähnten Verhalten der Aetherlösung keine weitere Blaufärbung mehr hervor.

Da man bei der Analyse der Chromverbindungen ron der Ueberchromsäure-Reaction meist nur in dem Falle Gebrauch machen wird, wo es sich um den Nachweis sehr geringer Mengen von Chromsäure handelt, und, wie sich aus dem Vorhergehenden ergeben dürfte, der Einfluss der Vanadinsäure auf die Empfindlichkeit, beziehungsweise das Zustandekommen der Wasserstoffsuperoxyd-Reaction überhaupt der augenscheinlichste ist, so ergibt sich ohne Weiteres die Frage, wie wohl am geeignetsten die Beeinträchtigung durch die erwähnte Säure beseitigt. werden kann.

Es ist mir gelungen auf Grund nachstehender Erwägungen den hinderlichen Einfluss der Vanadinsäure auf die Ueberchromsäure-Reaction aufzuheben. Bekanntlich besitzen mehrere anorganische Säuren die Eigenschaft, sich mit anderen Säuren, namentlich Phosphor- und Arsensäure zu Doppelverbindungen - sogenannten complexen Säuren - vereinigen zu können. $\mathrm{Zu}$ diesen Säuren gehören die Molybdänsäure, die Wolframsäure und auch die Vanadinsäure. Aus den erwähnten Umständen liess sich zwar nicht mit positiver Sicherheit behaupten, aber 
doch die Möglichkeit voraussehen, dass ein Zusatz von zum Beispiel Phosphorsäure, beziehungsweise phosphorsaurem Natron, durch Bildung einer Phosphorvanadinsäure die Einwirkung des Wasserstoffsuperoxyds auf Chromsäure sich ungestört vollziehen lässt. Dem ist in der That so, wie die nachfolgenden Ausführungen beweisen. Zum besseren Vergleich und zu genauerer Uebersicht und Beurtheilang wurden 3 Lösungen neben einander hergestellt, welche alle drei je $3 c c$ Kaliumbichromatlösung (entsprechend $0,003 g \mathrm{~K}_{2} \mathrm{Cr}_{2} \mathrm{O}_{7}$ ) und $3 \mathrm{cc}$ Wasserstoffsuperoxyd enthielten. Der Lösung I wurden ausserdem etwa $3 c c$ völlig gesättigter Natriumphosphatlösung $\left(\mathrm{Na}_{3} \mathrm{PO}_{4}\right)$ und $2 c c$ metavanadinsaures Ammoniak (entsprechend $0,002 \mathrm{~g}$ dieses Salzes) hinzugefügt. Lösung II enthielt ebenfalls $2 c c$ metavanadinsaures Ammoniak, während der Iösung III weder letzteres noch Natriumphosphat zugesetzt wurde. Mit einigen Tropfen concentrirter Salpetersäure wurden die 3 Lösungen sodann angesäuert und umgeschüttelt. Das Resultat war folgendes: Jede der drei Flüssigkeiten färbte sich gleichmässig blau. Während Lösung I und III allmählich in hellblaue Flüssigkeiten übergingen und diesen Farbentom längere Zeit beibehielten, färbte sich Lösung II sehr bald kräftig hellbraun und markirte einen auffallenden Gegensatz zu den beiden anderen. Auch als der Versuch in der Weise wiederholt wurde, dass Lösung II nur $1 c c$, ja nur $0,5 c c$ vanadinsaures Ammoniak enthielt, blieb die Gesammtwirkung genau dieselbe. Obwohl in letzterem Falle die Lösung I doppelte und vierfache Mengen von metavanadinsaurem Ammoniak enthielt, zeigte sie doch keine Neigung; irgendwie einen bräunlichen Farbenton anzunehmen. $\mathrm{Ob}$ die Entstehung von complexen Phosphorvanadinsäuren in der That den Grund zu dem merkwürdigen Verhalten der Vanadinsäure gegen die Ueberchromsäure bei Gegenwart von phosphorsaurem Natron bildet, mag vorläufig dahin gestellt bleiben. Für die Zwecke der Analyse ist das wohl ziemlich gleichgültig. Interessanter war die Frage, wie sich die Arsensäure, beziehungsweise inre Alkalisalze, unter den mitgetheilten Bedingungen verhalten würden. Werden die Versuche in genau derselben Art und Weise unter den gleichen Concentrationsbedingungen, wie vorher beschrieben, uusgeführt, nur mit dem Unterschiede, dass man an Stelle des phosphorsauren Natrons eine concentrirte Lösung von Arsensäure (2 $c c$ ) anwendet, so beobachtet man, dass bereits durch das Hinzufügen der freien Arsensäure zine tief dunkelblaue Färbung eintritt; letztere verschwindet aber sehr schnell wieder, und sowohl Lösung I wie II nehmen eine bräunliche Farbe an. 
Gänzlich verschieden von der freien Arsensäure verhält sich das Natronsalz derselben $\left(\mathrm{Na}_{3} \mathrm{As}_{4}\right)$. Auch hier wurden der möglichst zu erleichternden Uebersicht wegen drei einander an Concentration und so weiter genau entsprechende Lösungen hergestellt, von denen die Lösung I ausser je $3 c c$ Kaliumbichromatlösung $\left(=0,003 g \mathrm{~K}_{2} \mathrm{Cr}_{2} \mathrm{O}_{7}\right)$ nnd Wasserstoffsuperoxyd $2 c c$ metavanadinsaures Ammoniak $(=0,002 g$ des letzteren Salzes) und etwa $3 c c$ einer concentrirten Lösung von Natriumarseniat enthielt. In Lösung II befand sich die gleiche Menge von vanadinsaurem Ammoniak ohne arsensaures Natron. Lösung III endlich enthielt keines der beiden zuletzt erwähnten Reagentien. Durch Ansäuern mitSalpetersäure wurde in allen drei Flüssigkeiten eine tief dunkelblaue Färbung hervorgerufen. Lösung I nimmt rasch eine hellblaue Farbe an und bleibt so, ohne eine Spur von bräunlicher Farbe zu zeigen. Lösung II bildet einen auffallenden Contrast zu der ersteren, indem nach sehr kurzer Zeit ein kräftiger, bräunlicher Farbenton auftritt. Lösung III verhält sich in ilıren Farbenabstufungen ungefähr so wie Lösung 1 .

Zur ferneren Feststellung der Möglichkeit, dass auch andere anorganische Salze ähnlich dem Phosphate und Arseniate des Natriums gegen Vanadinsäure reagiren könnten, wurden die nachstehend benannten Salze einer näheren Untersuchung unterworfen, und zwar zunächst das Nitrat des Kaliums. Je $3 c c$ Kaliumbichromatlösung $\left(0,1 \% \mathrm{~K}_{2} \mathrm{Cr}_{2} \mathrm{O}_{7}\right)$ and Wasserstoffsuperoxyd wurden mit einigen Cubikcentimentern völlig gesättigter Kaliumnitratlösung geschüttelt, und dazu $2 c c$ der 0,1 procentigen Ammoniumvanadatlösung gefügt. Da gleichzeitig in derselben Art, wie oben beschrieben, Controlversuche mit Lösungen, welche kein salpetersaures Kalium enthielten, gemacht wurden, so konnte man über die Einwirkung des Kaliumnitrats nicht im Zweifel bleiben. Dieselbe muss als eine durchaus negative bezeichnet werden. Wiederholt man den Versuch mit salpetrigsaurem Natrium unter den nämlichen Verhältnissen, so erhält man, falls man eine nur schwache Lösung des Nitrits zugesetzt hat, anfänglich eine schwache Bläuung, die aber sehr schnell wieder verschwindet und einer bräunlichen Farbe Platz macht. Wendet man concentrirte Natriumnitritlösungen an, so erhält man beim Hinzufügen von Salpetersäure überhaupt keine Blaufärbung, auch aufangs nicht.

Durch Elektrolyse von schwefelsaurem Ammoniak erhält man bekanntlich das sogenannte überschwefelsaure Ammoniak (Ammonium persulfuricum); wird dieses Salz, welches mit Chlorwasserstoffsäure Chlor entwickelt, in concentrirter Lösung zu der mehrfach erwähnten Bichromat- 
mischung hinzugefügt, so tritt schon ohne Zusatz von Salpetersäure Blaufärbung ein, welche aber sehr bald in eine bräunliche Farbe über. geht. Einen Einfluss auf die Vanadinsäure übt das überschwefelsaure Ammoniak demnach nicht aus; eben so wenig ist das jodsaure Kalium im Stande, die Beeinträchtigung der Ueberchromsäure-Reaction durch Vanadinsäure zu beseitigen.

Werden $3 c c$ Kaliumbichromatlösung mit $3 c c$ Wasserstoffsuperoxyd geschüttelt und $1^{1 / 2} c c$ kalt gesättigter Urannitratlösung hinzugefügt, so entsteht sofort eine dunkelblaue Färbung der vereinigten Flüssigkeiten; unter Fintwicklung kleiner Bläschen (Sauerstoff!) nimmt die Lösung bald einen hellblaven Ton an oder wird farblos, und es bildet sich ein emulsionsartig-suspendirter Körper, welcher sich nach 5-10 Minuten als Niederschlag absetzt. Wird zugleich mit dem Urannitrat vanadinsaures Ammoniak (2cc) der Kaliumbichromatlösung hinzugefügt, so zeigen sich ganz dieselben Erscheinungen; die über dem gelblich erscheinenden Niederschlage stehende Flüssigkeit ist sehr schwach bläulich bis farblos.

Zum Schlusse wurden einige Versuche mit dem Natrium-, beziehungsweise Ammoniumsalze der Wolfram- und Molybdänsäure gemacht, und zwar aus dem Grunde, weil diese Säuren analog der Vanadinsäure complexe Säuren, besonders mit Arsen- und Phosphorsäure zu bilden vermögen, und möglicher Weise die Analogie mit der Vanadinsäure sich auch im Verhalten gegen die Ueberchromsäure äussern könnte.

Werden je 5 cc Wasserstoffsuperoxyd- und Kaliumbichromatlösung (entsprechend: $0,005 \mathrm{~g} \mathrm{~K} \mathrm{Kr}_{2} \mathrm{O}_{7}$ ) mit 1 Tropfen Salpetersäure angesäuert, so erhält man die bekannte tief dunkelblaue Färbung der Flüssigkeit; werden zu dieser letzteren einige Cnbikcentimeter einer concentrirten Lösung von molybdünsaurem Ammoniak hinzugefügt, so verschwindet die Blaufärbung sofort. Werden je $10 c c$ Kaliumbichromat- $\left(=0,01 g \mathrm{~K}_{2} \mathrm{Cr}_{2} \mathrm{O}_{7}\right)$ und Wasserstoffsuperoxydlösung mit 1 Tropfen Salpetersäure und $2 c c$ einer 0,1 procentigen Ammoniummolybdatlösung (entsprechend also $0,002 \mathrm{~g}$ molybdänsaurem Ammon) geschüttelt, so entsteht tief blaue Fürbung durch Bildung von Ueberchromsäure. Allmählich erfolgt Entfärbung, jedoch ist die Einwirkung solch' verdünnter Molybdänsäurelösungen keine besonders auffällige und geht nur langsam von Statten; jedenfalls wirkt eine Vanadinsäurelösung von gleicher Verdünnung im nämlichen Falle energischer und schneller auf die Ueberchromsäure.

Versetzt man je $10 \mathrm{cc}$ Wasserstoffsuperoxyd- und Kaliumbichromatlösung (entsprechend $0,001 \mathrm{~g} \mathrm{~K}_{2} \mathrm{Cr}_{2} \mathrm{O}_{7}$ ) mit etwas Salpetersäure und fügt 
586 Reichard: Ceber d. Nachw. d. Chromsäure dureh Wasserstoffsuperoxyd etc.

zu der tief dunkelblau gewordenen Flussigkeit $5 c e$ einer mässig concentrirten Lösung von wolframsaurem Natrium, so entsteht nach wenigen Augenblicken Entfärbung, das heisst nach Uebergang in hellblau tritt bald ein gelblich-bräunlicher Farbenton auf. Ist die blaue Farbe verschwuuden, und setzt man wieder etwas Kaliumbichromat zu, so tritt dadurch keine weitere Blaufärbung ein; eine solche erfolgt erst, wenn gleichzeitig auch Wasserstoffsuperoxyd hinzugefügt wird, doch entlärbt sich diese zweite blaue Flüssigkeit nach wenigen Augenblicken wieder.

Zusammenstellung der Ergebnisse der vorliegenden Abhandlung.

Kurz zusammengefasst lassen sich die Resultate dahin präcisiren:

1. dass die Vanadinsüure einen sehr bemerkenswerthen Einfluss auf das Zustandekommen der Ueberchromsäure-Reaction ausübt, und zwar indem sie letztere stark beeinträchtigt;

2. dass noch bei einem Verhältnisse von 10 Theilen Kaliumbichromat zu 1 Theil vanadinsaurem Ammoniak die Einwirkung der Vanadinsäure deutlich wahrnehmbar ist;

3. dass sowohl arsensaures als phosphorsaures Natron den Einfluss der Vanadinsäure auf Ueberchromsäure aufheben ${ }^{1}$ );

4. dass andere Salze, von denen das Nitrat und Nitrit des Natriums das ibersehwefelsaure Ammoniak, das jodsaure Kalium und das Urannitrat näher geprüft wurden, keinen bemerkenswerthen Einfluss auf das Verhalten der Vanadinsäure gegenüber Ueberchromsäure ausüben;

5 . dass durch die Anwesenheit von Säuren, welche der Vanadinsäure in gewisser Beziehung nahe stehen, (Molybdän- und Wolframsäure) in ähnlicher Weise die Ceberchromsäure-Reaction beeinträchtigt wird.

Beztiglich des Punktes 5 beabsichtige ich demnächst eine eingehendere Untersuchung zu veröffentlichen.

1) Es wird sich wohl empfehlen, dem phosphorsauren Natron vor dem arsensauren den Vorzug zu geben, da sich letztere Verbindung durch Bchwefelwasserstoff fallen lässt und unter Umständen vielleicht durch diese Figenschaft störend einzuwirken im Stande wäre. 